\title{
Novel and Efficient Dye-linked Photoinitiator generating a Radical via an Intramolecular Electron-transfer Process
}

\author{
Koichi Kawamura*, Takuma Amemiya ${ }^{\dagger}$, Yoshihiro Nakai ${ }^{\dagger}$, Masanobu Takashima ${ }^{\dagger}$ \\ *Synthetic Organic Chemistry Laboratories, FUJIFILM Corporation, 577 Ushijima, Kaisai, \\ Kanagawa, 258-857, Japan. 'Finechemicals Business Div., FUJIFILM Corporation, 2-12-1, \\ Ohgi, Odawara, Kanagawa, 250-000, Japan. "Life Science products, Div., FUJIFILM \\ Corporation, 9-7-3, Akasaka, Minato-ku, Tokyo, 107-0052, Japan.
}

\begin{abstract}
A merocyanine dye (D) has been attached covalently to a substituted bis(trichloromethyl)-1,3,5-triazine initiator (T) to give the dye-linked initiator (D-T) in order to reveal the effect of the linked systems on the efficiency of photopolymerization. Fluorescence spectroscopy indicates efficient intramolecular quenching of $\mathrm{D}$ emission by $\mathrm{T}$ in the dye-linked initiator (D-T) compared to intermolecular quenching in a simple mixture of the dye and the initiator $(\mathrm{D} / \mathrm{T})$. The relative photoinitiating efficiency of dye-linked initiators in photopolymerization of acrylates was also evaluated and D-T showed higher photosensitivity than the $\mathrm{D} / \mathrm{T}$ mixtures. The mechanism of radical generation of D-T was proposed and the effect of size of the generating radical was evaluated by a chain transfer agent.
\end{abstract}

Keyword: photoinitiator, intramolecular electron-transfer, dye-linked initiator, radical, visible light

\section{Introduction}

The search for efficient polymerization photoinitiating systems operating under visible light excitation has been made since such systems are expected to have wide applications in typical practical fields, such as high-speed laser imaging, 3D polymerization systems and photo-curable adhesive materials. Very few cleavable radical photoinitiators (Type I) working under visible light are known as yet. Accordingly, it is necessary to use two-component or multi-component photoinitiating systems. Improvement of these combinations has led, for example, to the design of photoinitiating systems such as dye/amine, dye/onium or dye/borate salt ion pairs. These processes involve the photo-excitation of a dye to initiate electron transfer, for example, from a donor (dye) to an acceptor (initiating part) molecule. Electron transfer processes in photopolymerization and imaging processes have recently been reviewed [1-8].
It is generally accepted that the rate of the electron transfer Ket depends not only on the free energy change but also on the distance between donor and acceptor. The relationship between Ket and donor-acceptor separation $\mathrm{R}_{\mathrm{DA}}$ has been well studied theoretically and experimentally, and indicates the sharp fall of Ket as donor and acceptor are separated by more than one or two classical collisional diameters [9-11]. Dye-linked photoinitiators, in which a radical generating part is brought close to a photosensitizing dye-chromophore by a covalent bond, have been shown to be very useful as free-radical sources [12-16].

In order to investigate the behavior of dye-linked initiator in more detail, we have prepared a merocyanine dye-linked photoinitiator (DT) and investigated its photochemical behaviors. Furthermore, the role of the new initiator in standard acrylate compositions was also examined. 


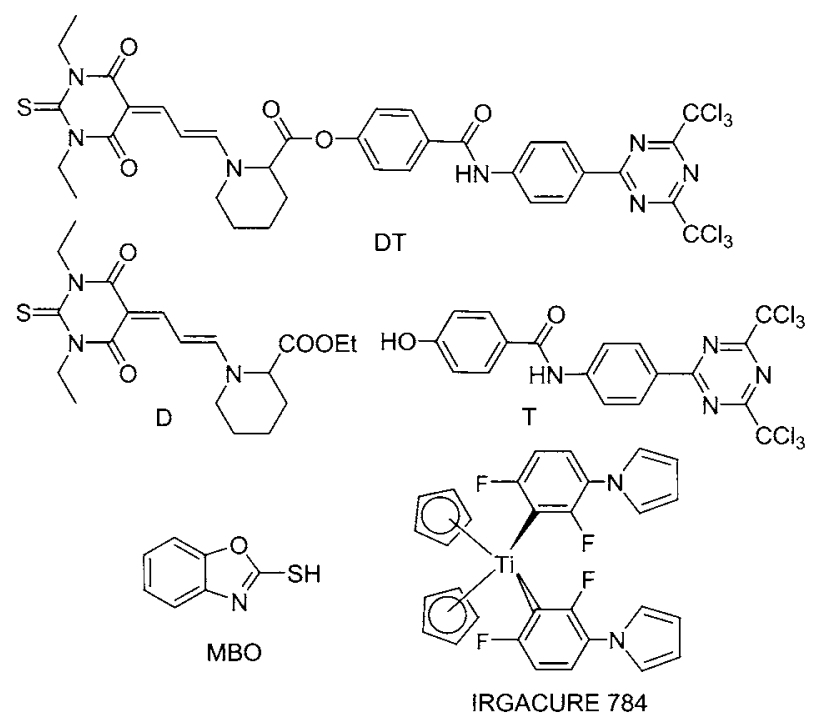

Figrure.1
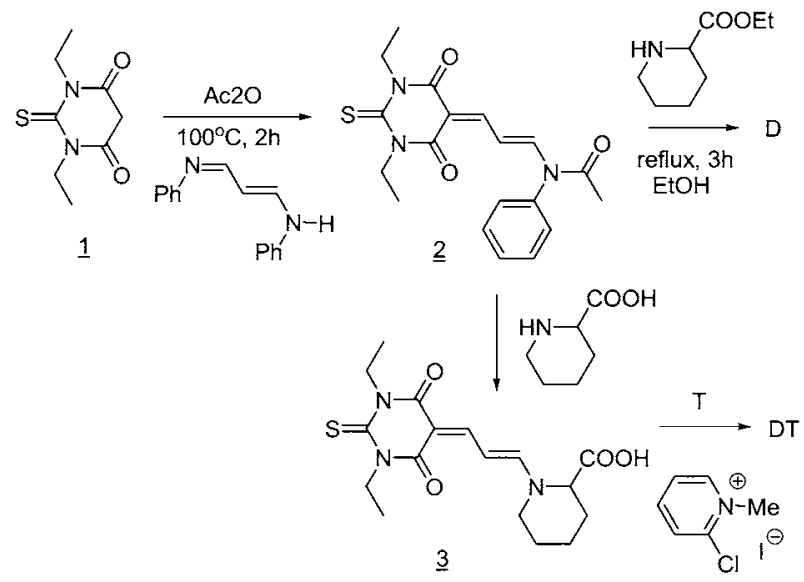

Scheme 1:

\section{Experimental}

2.1 General Methods

Steady-state absorption spectra in the UV-visible regions were measured on a UV2500 (Simazu) spectrophotometer. Fluorescence measurements were recorded in an atmosphere, using the absolute quantum yield measurement system (C9920-02, Hamamatsu Photonics) which measure the emission using a multi-channel analyzer capable of instantaneous measurement of the whole spectrum by employing a high sensitivity CCD sensor, thus avoiding the photodecomposition of the sample during fluorescence measurements. Fluorescence spectra of films were monitored with front-face detection on coatings with absorbance values not more than 0.15 absorbance units. Double-bond conversion was monitored by using a Nicolet FT-IR spectrophotometer.

\subsection{Film Preparation}

Films for the measurement of photophysical behavior and photopolymerization were typically prepared in the following manner. The photoinitiator (a merocyanine/triazine combination or a linked initiator), a polyfunctional acrylate monomer (Dipentaerythritol hexacrylate; DPHA, Shin-Nakamura Chemical) and a polymeric binder, (polymethyl methacrylate, PMMA, Mw $=15000$, Aldrich) were dissolved in a 64:4:4 mixture of solvent (Methylethyl ketone / 1-Methoxy-2propanol /Tetrahydrofuran, w/w). The weight ratio of PMMA/DPHA was 6.5/3.0 and the concentration of photoinitiator was $30 \mathrm{mmole}$ per $1 \mathrm{Kg}$ of total amount of PMMA and DPHA. The solution was cast on a glass or on a silicon wafer and the solvent was evaporated with heating at $80{ }^{\circ} \mathrm{C}$ for $5 \mathrm{~min}$. The thickness of the film was about $1 \mu \mathrm{m}$.

For experiments in section 3.7, which compare photosensitivity of the linked photoinitiator with the commercially available Irgacure 784, PMMA was eliminated and only two ingredients, DPHA and photoinitiator, were used.

\subsection{Measurement of Photosensitivity}

The light source was a high pressure mercury lamp (EXECURE3000, HOYA) and band-pass filters (BP440, Kenko Co., Ltd) were used to irradiate merocyanine alone $\left(\lambda=440 \mathrm{~nm}, 1.48 \mathrm{~mW} / \mathrm{cm}^{2}\right)$. The kinetics of the polymerization was measured by following the disappearance of the IR absorption of the acrylic double bond at 1409 $\mathrm{cm}^{-1}(\mathrm{CH} 2=\mathrm{CH}$ twisting, in plane $)$. The percentage of conversion was calculated from the decrease of the area of the IR absorption peak at 1400.1-1417.4 $\mathrm{cm}^{-1}$ of the sample after exposure. For comparison experiments with the commercially available Irgacure 784, the sharp cult filter (L39) which transmit light over $390 \mathrm{~nm}\left(40 \mathrm{~mW} / \mathrm{cm}^{2}\right)$ was used for practical reasons.

\subsection{Compound D}

A mixture of 2 (6.0 g, $16.1 \mathrm{mmol})$, which was prepared from 1,3-diethylthiobarubituric acid and $\mathrm{N}$-[3-(Phenylamino)-2-propenylidene]aniline in acetic anhydride as a condensing agent, and 2-carboethoxy piperidine $(2.79 \mathrm{~g}, 17.8 \mathrm{mmol})$ were heated under reflux in ethanol for $4 \mathrm{hr}$. Upon cooling to room temperature, the yellow flocculent was precipitated from the solution. The precipitate was filtered and recrystallized from ethanol. $2.5 \mathrm{~g}$; 
mp $190.3-192.1^{\circ} \mathrm{C} ; \lambda_{\max }=431.5 \mathrm{~nm}(\varepsilon=119700$ in THF).

\subsection{Compound DT}

To a solution of 2-Chloro-1-methylpyridinium iodide ( $2.94 \mathrm{~g}, 11.5 \mathrm{mmol}), \mathrm{T}(4.7 \mathrm{~g}, 8.2 \mathrm{mmol})$ and $\underline{\mathbf{3}}(3.0 \mathrm{~g}, 8.2 \mathrm{mmol})$, which was prepared from $\underline{\mathbf{2}}$ and 2-carboxyl piperidine as described above, in $60 \mathrm{~mL}$ of THF was added 4-( $N, N$-dimethylamino)pyridine $(0.1 \mathrm{~g}, 0.82 \mathrm{mmol})$ followed by dropwise addition of $2.3 \mathrm{ml}$ (16.4 mmol) of triethylamine with cooling. After stirring for 3.5 hour at room temperature, the reaction mixture was poured into water and extracted with ethyl acetate. Flash column chromatography on silica gel with hexane/ethyl acetate gave DT (3.19 $\mathrm{g}, 44 \%$ ) as yellow crystal ; $\mathrm{mp} 184.0-185.2^{\circ} \mathrm{C}$; $\lambda_{\max }=432.0 \mathrm{~nm}(\varepsilon=118300 \mathrm{in} \mathrm{THF})$

\section{Results and Discussion}

\subsection{Steady-state Absorption Spectra and Fluorescence spectra}

The absorption spectra of the initiator compounds in the matrix film (PMMA/DPHA) are shown in Figure 2. The absorption maxima of linked compounds (DT) around $350 \mathrm{~nm}$ and $430 \mathrm{~nm}$ are attributed to triazine $(\mathrm{T})$ and merocyanine dye (D) respectively and almost identical with that of an equimolar mixture of each component merocyanine and triazine $(\mathrm{D}+\mathrm{T})$. However slightly longer shift is observer in DT compared to D (DT: $434 \mathrm{~nm}, \mathrm{D}: 433 \mathrm{~nm})$. We presume this shift indicates the effective intramolecular interaction between dye chromophore and traizine chromophore, because the same longer shift $(1 \mathrm{~nm})$ of $D$ is observed where large excess (10 folds) of traizine (T) is present in the D+T system.

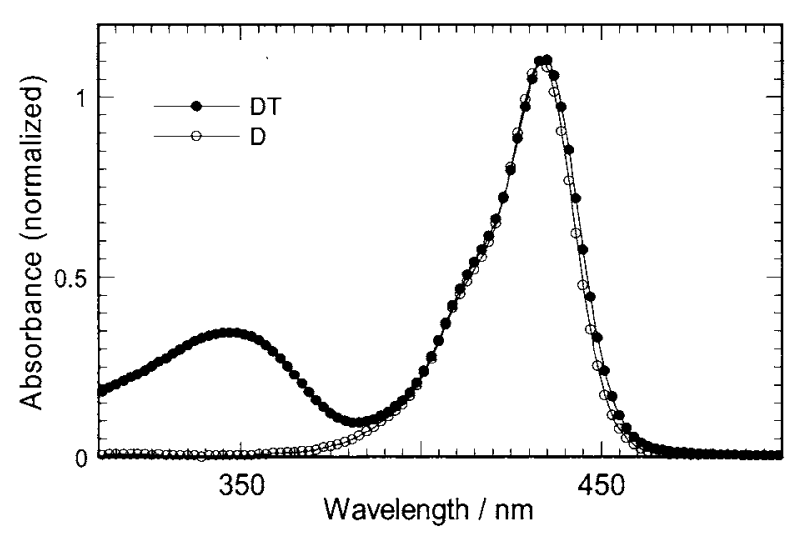

Figure 2. The absorption spectra of DT and D in the matrix film (PMMA/DPHA).

\subsection{Fluorescence Quenching Experiments}

The fluorescence spectra of the merocyanine and the linked compounds in the matrix film (PMMA/DPHA) are shown in Figure 3. The fluorescence of dye chromophore in DT is quenched by traizine chromophore, indicating intramolecular electronic interaction in the excited state. The fraction of quenching (quenching efficiency \%) is regarded as a measure of efficiency of intramolecular interaction between the merocyanine and the triazine parts. It has become apparent from Figure 3 that the quenching efficiency of DT is about $50 \%$, that is, $50 \%$ of excited dye chromophore is quenched intramolecularly by triazine chromophore.

It is important to compare the intramolecular quenching with the intermolecular ones. Thus, the latter was evaluated by measuring fluorescence of dye in a simple mixture of $\mathrm{D}$ and $\mathrm{T}$ in the photopolymer film. It was revealed that the fluorescence was quenched intermolecularly to less than $5 \%$ by an equimolar amount $\left(10 \mathrm{mmol} / \mathrm{dm}^{3}\right)$ of triazine. This means intramolecular quenching in DT is very efficient compared with intermolecular quenching.

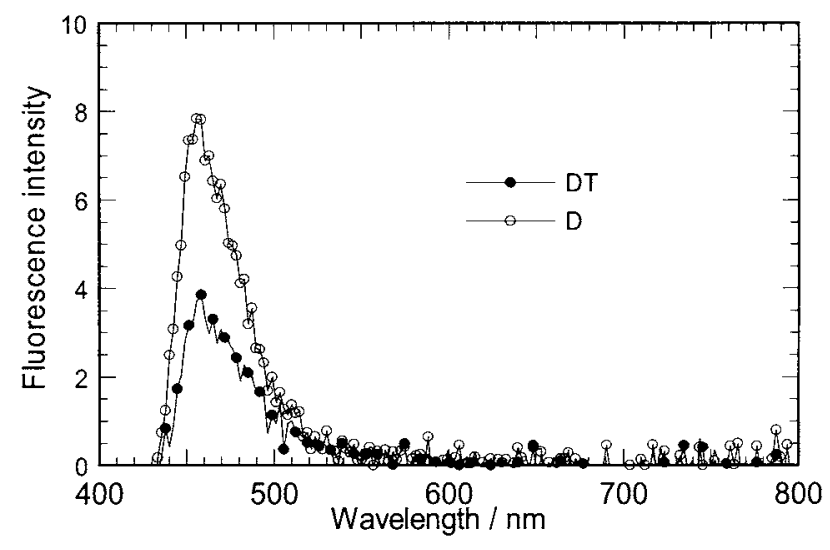

Figure 3. The fluorescence spectra of the merocyanine and the linked compounds in the matrix film (PMMA/DPHA). Excitation: $420 \mathrm{~nm}$. Concentration of $\mathrm{D}$ and DT are $10 \mathrm{mmole} / \mathrm{dm}^{3}$

\subsection{Efficiencies of Photopolymerization}

The spectroscopic studies clearly showed that merocyanine-linked initiators exhibit a marked increase in the efficiency of fluorescence quenching than a simple mixture of a dye and an initiator. These results are reasonably explained in terms of the efficiency of electron transfer between a dye and an initiator, which eventually results in 
the formation of a radical. Thus it is very important to examine whether a similar trend can be observed in a practical photopolymerization systems.

Thus, the relative efficiency of merocyanine-linked triazine as an initiator in photoinitiating systems of acrylates monomers was evaluated by monitoring the disappearance of the acrylic double bond in the IR absorption. Figure 4 shows the kinetics of polymerization employing merocyanine-linked photoinitiator under irradiation. The results clearly show that the rate is enhanced when the linked photoinitiator DT is used, as opposed to the unlinked photoinitiator combinations $(\mathrm{D} / \mathrm{T})$ as suggested by fluorescence quenching.

It is noteworthy that the merocyanine dye (D) alone can cause polymerization, that is, $\mathrm{D}$ can work as a photoinitiator. This is in contrast to a simple merocyanine dye which is stable under photoirradiation and does not work as a photoinitiator as we have reported before [14]. From these results, it was found that the dye radical is formed in addition to traizine radical and contributes in photopolymerization in $\mathrm{DT}$ and $\mathrm{D} / \mathrm{T}$ systems.

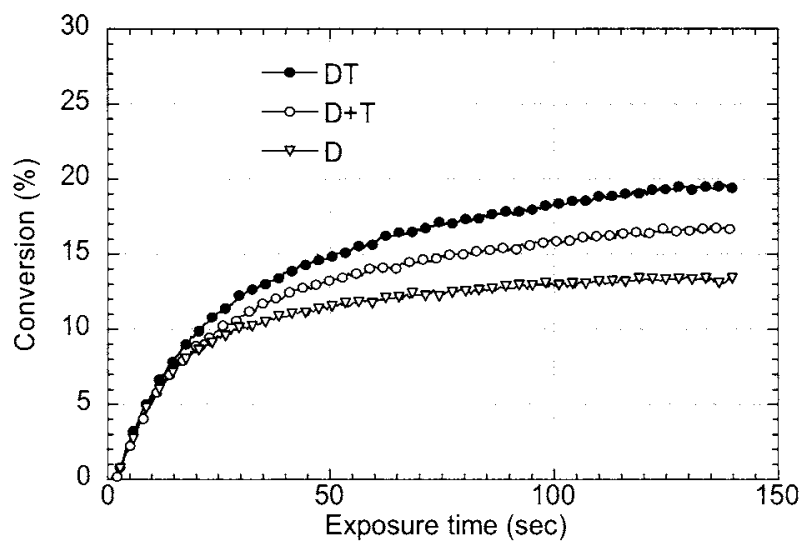

Figure 4. Conversion ( $\%$ ) of acrylate double bonds as a function of time for polymerization of acrylates containing photoinitiators on irradiation $(\lambda=440 \mathrm{~nm}$, $\left.1.48 \mathrm{~mW} / \mathrm{cm}^{2}\right)$. Photoinitiating system $\left(30 \mathrm{mmol} / \mathrm{dm}^{3}\right)$; $\mathrm{DT}(\bullet), \mathrm{M}+\mathrm{T}(\mathrm{O})$ and $\mathrm{D}(\Delta)$

\subsection{Steady-state Photolysis}

The spectral change observed by steady-light irradiation of DT and D in photopolymer coatings are shown in Figure 5 (a) and (b). Photobleaching was observed not only for DT (Figure 5 a) and D/T system (not shown here) but also for $\mathrm{D}$ alone (Figure $5 \mathrm{~b}$ ), although photobleaching was apparently slightly efficient in DT. The photobleaching of D suggests the photoinitiating ability of $\mathrm{D}$ molecule, that is, $\mathrm{D}$ can generate initiating radicals under photoirradiation.
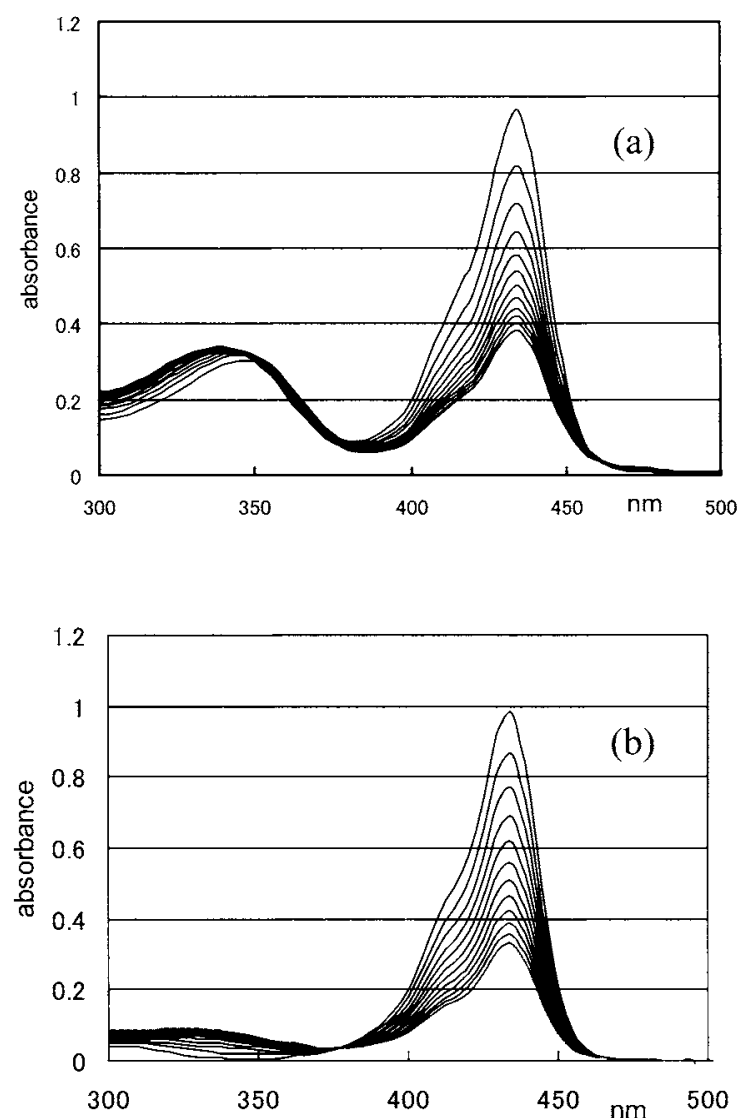

Figure 5. Change of absorption spectra of DT (a) and D (b) in the photopolymer coatings $\left(30 \mathrm{mmol} / \mathrm{dm}^{3}\right)$, upon irradiation at $440 \mathrm{~nm}$. Each spectrum was recorded in every $10 \mathrm{sec}(1.84 \mathrm{~mW} / \mathrm{cm} 2)$.

\subsection{Possible Mechanism of Linked Photoinitiating system}

We have reported the mechanism of a radical generation of triazine (T) in the presence of simple merocyanine dyes (M) [14]. In view of the analogues dye/triazine photosensitization mechanism, it is reasonable to assume that intramolecular electron transfer take place from excited dye moiety to triazine moiety to generate merocyanine cation radical $\left(\mathrm{D}^{+}\right)$/triazine anion radical $\left(\mathrm{T}^{-}\right)$pairs. The latter then could form a chloride anion and a triazine radical, which enters into the polymerization reaction as shown in Figure 6 (path a). This mechanism is reasonable from the analogous dye/ triazine photosensitization reaction which was reported by Scaiano et al [17]. 
In contrast to our previous report, DT would have the another radical generating path as shown in Figure 6 (path b). That is, DT decomposes directly upon irradiation to form radials from dye moiety without quenching by triazine as suggested by induction of photopolymerization by $\mathrm{D}$ molecule alone.

The detailed radical generation mechanism from $\mathrm{D}$ is not clear at present, but carbon-hydrogen atom adjacent to nitrogen atom of piperidin ring in D would be susceptible to hydrogen abstraction to cause polymerization in view of $\mathrm{N}$ containing molecules like triethanolamine or $\mathrm{N}$-phenylglycine having adjacent $\mathrm{C}-\mathrm{H}$ bond work as radical generating agents by hydrogen abstraction.

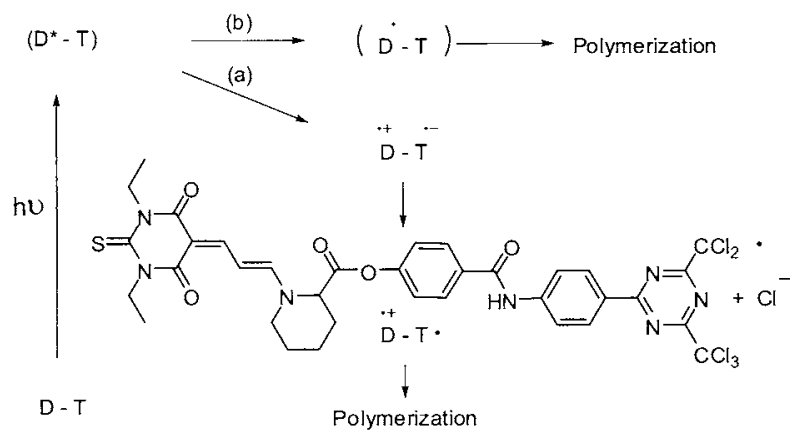

Figure 6. Proposed mechanism of radical generation in the photopolymer film.

3.6 Enhancements of Photopolymerization by Chain transfer agent

It is generally accepted that the diffusion of large radicals is controlled by the viscosity of the surrounding medium and would be severely limited in highly viscous media such as photopolymer coatings. The radical generated from DT is large radical as suggested in Figure 6; therefore it would be susceptible to the diffusion effect. We presume that this would be the one of the reasons why the polymerization tends to saturate with irradiation as shown in Figure 4.

As a general rule, in order to increase polymerization by avoiding the diffusion limited reaction of bulky radicals, thiol compounds having low molecular weight, such as 2-mercaptobenzoxazole (MBO), are added as chain transfer agents. Hydrogen abstraction would take place from bulky radicals (D-T) to 2-mercaptobenzoxazole (MBO) to form small reactive radicals, which further give rise to polymerization.
Thus, 2-mercaptobenzoxazole (MBO, 10 fold excess) was added in the photopolymer film containing initiators (DT or $\mathrm{D} / \mathrm{T}$ ) and conversion of acrylate was compared as shown in Figure 7. It is apparent from this figure that the conversion at $200 \mathrm{~mJ} / \mathrm{cm}^{2}$ is increased by 1.4 times by the addition of MBO in DT, whereas the increment is smaller (1.2 times) in $\mathrm{D} / \mathrm{T}$, indicating the chain transfer agent works more effectively for bulky radicals derived from linked initiator.

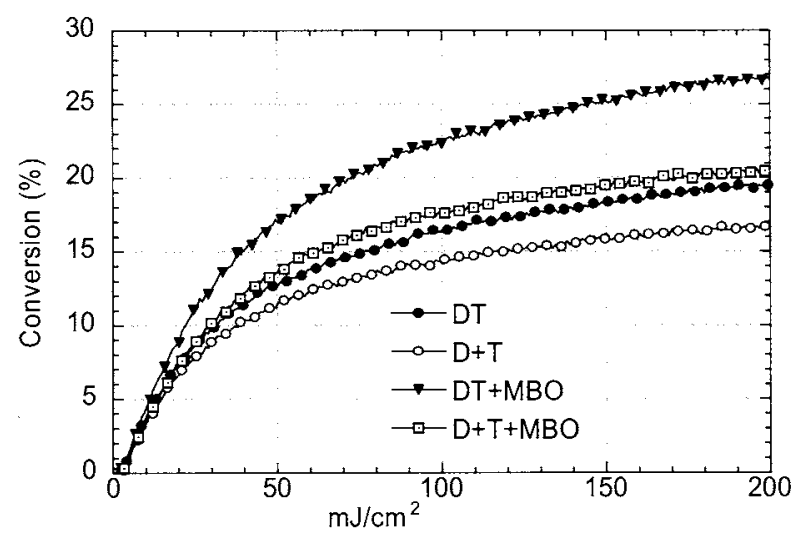

Figure 7. Conversion (\%) of acrylate double bonds as a function of time for polymerization of acrylates containing photoinitiators $\left(30 \mathrm{mmol} / \mathrm{dm}^{3}\right)$ and $\mathrm{MBO}$ $\left(300 \mathrm{mmol} / \mathrm{dm}^{3}\right)$ on irradiation $(\lambda=440 \mathrm{~nm})$.

3.7 Comparison of Linked Photoinitiating system with commercial photoinitiators

It is important here to compare the photosensitivity of linked photoinitiator (DT) with that of commercially available initiator. IRGACURE 784 is a typical visible light photoinitiator having absorption extending to about $500 \mathrm{~nm}\left(\lambda_{\max }=396 \mathrm{~nm}, \varepsilon=1380\right.$ in THF $)$. Conversion of acrylate was compared in photopolymer film as shown in Figure 8, where DT and IRG784 was used as photoinitiator and DPHA was used as a single matrix component. Irradiation wavelength was adjusted to cover all region of the visible light using $390 \mathrm{~nm}$ cut-off filter which would be commonly used condition for evaluating polymerization in industries.

It is revealed that DT is more effective than IRG784 in the same concentration $\left(30 \mathrm{mmol} / \mathrm{dm}^{3}\right)$ or even in a dilute condition DT $\left(15 \mathrm{mmmol} / \mathrm{dm}^{3}\right)$ shows higher photosensitivity than IRG784 $\left(90 \mathrm{mmol} / \mathrm{dm}^{3}\right)$. This means lesser amount is enough for DT than IRG784 to achieve the same photocuring, indicating more advantages of DT from the industrial standpoint. 


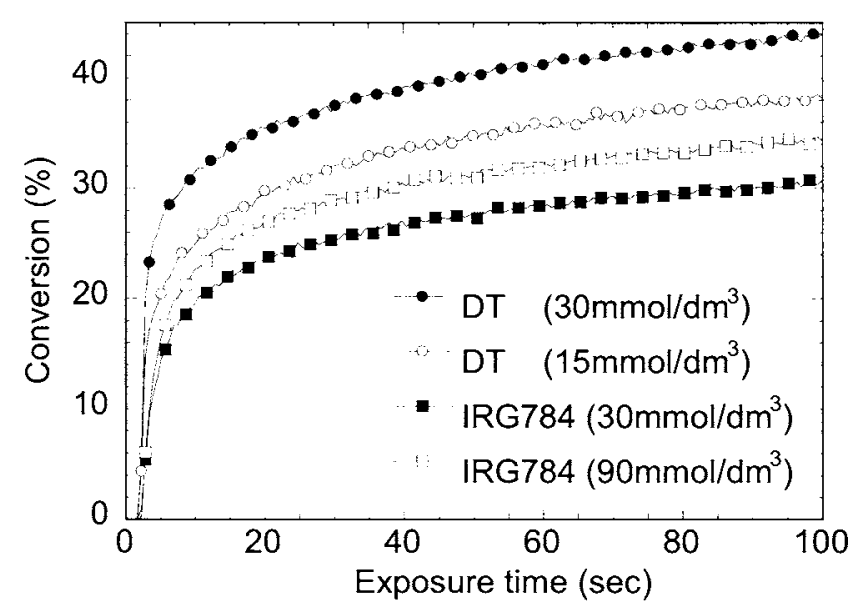

Figure 8. Conversion ( $\%$ ) of acrylate double bonds as a function of time for polymerization of acrylates containing photoinitiators (DT or IRG784)on irradiation $\left(\lambda>390 \mathrm{~nm}, 40 \mathrm{~mW} / \mathrm{cm}^{2}\right.$ at $\left.405 \mathrm{~nm}\right)$.

\section{Conclusions}

The photochemical processes of DT were examined in a photopolymer matrix and compared with that of $\mathrm{D} / \mathrm{T}$ and $\mathrm{D}$. The spectroscopic studies clearly showed that the dye-linked initiator (DT) exhibit a marked increase in the efficiency of fluorescence quenching compared to a simple mixture of the dye /initiator $(\mathrm{D} / \mathrm{T})$. The relative photoinitiating efficiency of dye-linked initiator in photopolymerization of acrylates was also evaluated and DT showed higher photosensitivity than the $\mathrm{D} / \mathrm{T}$ mixtures. A chain transfer agent was found to be effective to increase reactivity of linked initiator (DT) and DT is more effective than the commercial IRG784 even in a dilute condition.

\section{Acknowledgements}

We thank Mr. Hiroshi Yokoyama at Analysis Technology Center in FUJIFILM Corporation for fluorescence measurements.

\section{References and Notes}

1. J.P. Fouassier, Recent Res. Devel. Polymer Science 4 (2000) 131.

2. B.M. Monroe, G.C. Weed, Chem. Rev. 93 (1993) 435.

3. J.P. Fouassier, (Eds.), "Photochemistry and UV curing; New Trends 2006" Research Signpost, Kerala, India (2006).

4. R.S. Davidson, J. Photochem. Photobiol. A: Chem. 73 (1993) 81.

5. R.S. Davidson, in: J.P. Fouassier, J.F. Rabek (Eds.), "Radiation curing in polymer science and technology", Elsevier applied science, London and New York, Vol. 3, 1993, pp. 153.

6. J. Kabatc, M. Zasada, J. Paczkowski, J. Polym. Sci. Part A Polym. Chem. 45 (2007) 3626.

7. M. EL-Roz, J. Lalevee, F. M-Savary, X. Allonas, J. P. Fouassier, J. Polym. Sci. Part A Polym. Chem. 46 (2008) 7369.

8. D. Kim, J. W. Stansbury, J. Polym. Sci. Part A Polym. Chem. 47 (2009) 887.

9. N.J. Turro, in: "Modern Molecular Photochemistry", The Benjamin/ Cummings Publishing, California, 1978, pp. 316- 321.

10. G.J. Kavarnos, N.J. Turro, Chem. Rev. 86 (1986) 401.

11. H. Oevering, M. N. Paddon-Row, M. Heppener, A.M. Oliver, E. Cotsaris, J.W. Verhoeven, N.S. Hush, J. Am. Chem. Soc. 109 (1987) 3258 .

12. K. Kawamura, Japan Patent 2720195, 1990 , U.S. Patent 5262276, 1993, Chem. Abst. 112 (1990) 207938.

13. K. Kawamura, Y. Aotani, H. Tomioka, J. Physical Chemistry, B, (2003), 107, 4579.

14. K. Kawamura, J. Photochem. Photobiology A: Chemistry, 162 (2004) 329.

15. D. Burget, F. Amat-Guerri, J.P. Fouassier, R. Mallavia, R. Satre, Acta Polym. 50 (1999) 337.

16. A. Fimia, R. Sastre, F. Amat-Guerri, R. Mallavia, Macromolecules 27 (1994) 2643.

17. G. Pohlers, J.C. Scaiano, R. Sinta, R. Brainard, D. Pai, Chem. Mater. 9 (1997) 1353. 